\title{
Epidemiology of Upper Gastrointestinal Hemorrhage in the USA: Is the Bleeding Slowing Down?
}

\author{
Marwan S. Abougergi ${ }^{1,2}$
}

Published online: 3 February 2018

(c) Springer Science+Business Media, LLC, part of Springer Nature 2018

Upper gastrointestinal hemorrhage is a frequent gastrointestinal emergency accounting for $>250,000$ hospitalizations annually in the USA [1]. Traditionally, upper gastrointestinal hemorrhage has been divided into non-variceal and variceal, due to the significantly different prognosis associated with each category. The last two decades have witnessed major advances in upper gastrointestinal hemorrhage preventive and treatment modalities, including the discovery of Helicobacter pylori, the widespread use of proton pump inhibitors, and the development of novel endoscopic and radiologic hemostatic techniques. Countering these positive developments during the same time period has been the unprecedented widespread use of antiplatelet drugs and anticoagulants, [2] in addition to an epidemic of obesity and opioid abuse, all of which are risk factors for variceal and non-variceal upper gastrointestinal hemorrhage.

Multiple studies have attempted to describe the impact of those changes on the epidemiology and treatment outcomes of patients with upper gastrointestinal hemorrhage [1, 3, 4]. Based on small studies conducted at tertiary referral centers, upper gastrointestinal hemorrhage mortality was reported until recently to be constant for the past five decades. With the availability of large and powerful national databases in the USA, more accurate estimates became possible and confirmed what has long been suspected: that both variceal and non-variceal gastrointestinal hemorrhage mortality has actually been decreasing over the last several decades [1, 3, 4]. In this issue of Digestive Diseases and Sciences, Wuerth et al. report their results of a longitudinal retrospective database analysis that used the Nationwide Inpatient Sample (NIS) for calendar years 2002-2012 [5]. The NIS

Marwan S. Abougergi

mabougergi@catalystsclinic.com

1 Catalyst Medical Consulting, Simpsonville, SC, USA

2 Division of Gastroenterology, Department of Internal Medicine, University of South Carolina School of Medicine, Columbia, SC, USA is the largest publically available all-payer inpatient care database in the USA, nationally representative and yielding accurate national estimates of disease burden and healthcare resource utilization. It contains discharge data from more than 4000 short-term and non-Federal hospitals each year, yielding information on $\sim 7$ to 8 million hospital stays each year. The NIS data can be used to identify, track, and analyze national trends in health care utilization, access, charges, quality, and outcomes. NIS data are available from 1988 through the present, thereby facilitating analysis of trends over time. The number of states participating in the NIS has grown from 8 at its inception to 46 at present [6].

In this study, Wuerth et al. include adult participants admitted to the hospital urgently and/or emergently that were not transferred to another hospital and had a principal discharge diagnosis of acute upper gastrointestinal hemorrhage. The outcomes were temporal trends in the hospitalization rate and all-cause in-hospital mortality rate of each hemorrhage etiology. The authors report on the 2,432,088 discharges who met the study inclusion criteria from 2002 to 2012. The hospitalization rate of upper gastrointestinal hemorrhage in the USA decreased from 81 to $67 / 100,000$ population from 2002 to 2012 , a $21 \%$ decrease. This declining trend outpaced that of patients with diagnoses other than upper gastrointestinal hemorrhage $(1 \%, p<0.01)$. Peptic ulcer disease was the most common cause of hemorrhage across the years; the second most common cause was gastritis in 2002 and esophagitis in 2012. The third most common cause was esophagitis in 2002 and gastritis in 2012. From 2002 to 2012, the hospitalization rate of peptic ulcer hemorrhage decreased by $30 \%$, whereas that of Dieulafoy lesions, angiodysplasia, and neoplasm increased by 33,32 , and 50\%, respectively. The rates of esophageal variceal hemorrhage and Mallory-Weiss tears were constant. Similar to its incidence, the all-cause upper gastrointestinal hemorrhage case fatality rate decreased by $28 \%$, from $2.6 \%$ in 2002 to $1.9 \%$ in 2012 , a rate which paralleled that of patients with diagnoses other than upper gastrointestinal hemorrhage (23\% decrease, 
$p=0.19)$. The mortality rate for all of the different etiologic categories of non-variceal upper gastrointestinal hemorrhage declined over the study period. Yet, no mortality difference was reported for esophageal variceal hemorrhage over time. The largest reductions in mortality occurred for the diagnoses of esophagitis, neoplasm, and Mallory-Weiss tear (decreases of 39, 36, and 36\%, respectively).

So what does all this mean? What message can the gastroenterology and medical community take away from this study? In the words of Heraclitus, "the only thing that is constant is change." Updating the epidemiology and redefining the scope and the characteristics of patients affected by upper gastrointestinal hemorrhage is the first step toward maintaining the level of healthcare system preparedness needed to successfully prevent and treat upper gastrointestinal hemorrhage. Despite the inherent methodological limitations that are part and parcel of any administrative database analysis, Wuerth et al. present a number of important findings that deserve further discussion and that can generate additional questions for future study. First, it is noteworthy that the incidence and in-hospital mortality rate associated with upper gastrointestinal hemorrhage have declined from 2002 to 2012. This finding complements and extends that of earlier studies, $[1,3,4]$ confirming that both the incidence and in-hospital mortality of upper gastrointestinal hemorrhage have been steadily declining since 1989 . This finding is especially important in the context of the everincreasing use of dual and triple antiplatelet aggregation therapy that followed the introduction of drug-eluting coronary artery stents in the early 2000s. Second, the decline in upper gastrointestinal hemorrhage in-hospital mortality paralleled that of all other patients combined, a result in contrast to that of previous studies [1] that examined earlier time frames (1989-2009). This finding can potentially be explained by the hypothesis that the reduction in mortality rates was initially due to improvements in treatment modalities specifically offered for patients with upper gastrointestinal hemorrhage (hemostatic use of upper endoscopy with endoscopic therapy, proton pump inhibitors/octreotide and radiologic or surgical interventions) and now is dependent on advances in general healthcare delivery (hospital access, intensive care units, improved diagnostic testing, etc.). Third, the most common cause of non-variceal gastrointestinal hemorrhage is still peptic ulcer disease. Traditionally, non-variceal gastrointestinal hemorrhage treatment has been equated with peptic ulcer disease treatment, even in international guidelines [7]. The authors demonstrate that this assumption is still valid. As a result, gastroenterology training programs should continue to insure adequate trainee exposure to patients with peptic ulcer disease and require proof-of-proficiency in endoscopic ulcer treatment and hemostasis as a requirement for fellowship completion. Most importantly, with the widespread use of antiplatelet agents and anticoagulants that can cause and complicate peptic ulcer hemorrhage, more research is needed to provide an evidence-based answer to the question regarding when to restart those medications after endoscopic hemostasis is achieved. Fourth, the incidence of Dieulafoy lesions, angiodysplasia and neoplasm as etiology of upper gastrointestinal hemorrhage has been significantly increasing. While multiple advances in the treatment of peptic ulcer hemorrhage have been achieved over the past decade, including unipolar and bipolar heater probes, intravenous proton pump inhibitors and rotating hemostatic clips, little innovation has been introduced to the treatment of angiodysplasia- and neoplasm-induced hemorrhage. New modalities including single- and double-balloon enteroscopes that enable deep access to the small intestine, argon plasma coagulation and the promising early trials of TC-325 (Hemospray ${ }^{\circledR}$ ) have all stood shy of achieving long-lasting hemostasis in this patient population. Since recurrent hemorrhage in this situation remains an important problem, new medical and endoscopic therapeutic modalities will be needed to improve long-term patient outcomes as these hemorrhage etiologies become more common. Fifth, Wuerth et al. did not address the cost associated with the observed decrease in mortality rates over the past decade, another limitation. With the current healthcare environment focusing on cost-control as much as on quality-of-care, understanding the cost associated with the decrease in mortality and incidence of upper gastrointestinal hemorrhage is key to predicting its sustainability under the new reimbursement models. Another limitation is the probable under-coding of esophageal/gastric variceal hemorrhage in NIS. As the authors correctly point out in the article, variceal hemorrhage may have been coded as hematemesis without a specific diagnosis in cases where varices were not actively bleeding but had high-risk signs such as large varices, or the "red whale" or "white nipple" signs. This limitation is common to all administrative databases and might be overcome in future studies using nonadministrative data. Nevertheless, the many strengths of NIS, including its scope, unprecedented size, and its national representation compensate for this minor limitation, adding to the authors' insight and enabling generalizability to the entire USA. These are only some of the questions and stimulating findings that arise from this well-designed study. I, for one, will be on the lookout for the answers.

\section{References}

1. Abougergi MS, Travis AC, Saltzman JR. The in-hospital mortality rate for upper GI hemorrhage has decreased over 2 decades in the United States: a nationwide analysis. Gastrointest Endosc. 2015;81:882-888.

2. Brinker AD, Swartz L. Growth in clopidogrel-aspirin combination therapy. Ann Pharmacother. 2006;40:1212-1213. 
3. Taefi A, Cho WK, Nouraie M. Decreasing trend of upper gastrointestinal bleeding mortality risk over three decades. Dig Dis Sci. 2013;58:2940-2948.

4. Laine L, Yang H, Chang SC, et al. Trends for incidence of hospitalization and death due to GI complications in the United States from 2001 to 2009. Am J Gastroenterol. 2012;107:1190.

5. Wuerth BA, Rockey DC. Changing epidemiology of upper gastrointestinal hemorrhage in the last decade: a nationwide analysis. Dig Dis Sci. (Epub ahead of print). https://doi.org/10.1007/s1062 0-017-4882-6.
6. Healthcare cost and utilization project Web site. Available at: http://www.hcup-us.ahrq.gov/nisoverview.jsp. Accessed January 5, 2018.

7. Barkun AN, Bardou M, Kuipers EJ, et al. International consensus recommendations on the management of patients with nonvariceal upper gastrointestinal bleeding. Ann Intern Med. 2010;152:101-113. 